\title{
Metodología flipped classroom en la enseñanza universitaria
}

\author{
Pedro Canales-Ronda y Asunción Hernández-Fernández
}

\section{RESUMEN}

Pese a los cambios acontecidos en los hábitos y comportamiento de los estudiantes del siglo XXI en términos de interactividad y flexibilidad, en el ámbito universitario sigue predominando la metodología tradicional de clase magistral, por lo que es necesario profundizar tanto en la aplicación de nuevas metodologías como en la valoración de los alumnos sobre la utilidad de las mismas. En este contexto, el objetivo principal de este trabajo consiste en analizar la aplicación de la metodología flipped classroom. Para poder alcanzar el objetivo previsto, se ha trabajado con diferentes grupos de alumnos a través de dos experiencias, role playing y concurso. Los resultados obtenidos, a partir de un cuestionario ad-hoc, nos han permitido observar efectos positivos directos que han afectado a la mejora en la percepción del aprendizaje y de la satisfacción del alumnado de las asignaturas donde se puso en marcha, también esta metodología ha reforzado el trabajo en equipo dentro de un clima distendido y motivador, incrementando el dinamismo y la interacción en el aula.

Palabras clave: métodos de aprendizaje, innovación docente, trabajo en equipo, aprendizaje activo, motivación, universidad, España.

\section{Pedro Canales-Ronda}




\title{
Metodología flipped classroom no ensino universitário
}

\section{RESUMO}

Apesar das mudanças acontecidas nos hábitos e comportamento dos estudantes do século XXI em termos de interatividade e flexibilidade, no âmbito universitário continua predominando a metodologia tradicional de classe magistral, assim, é necessário aprofundar tanto na aplicação de novas metodologias como na avaliação dos alunos sobre a utilidade das mesmas. Neste contexto, o objetivo principal deste trabalho consiste em analisar a aplicação da metodologia flipped classroom. Para poder atingir o objetivo previsto, se trabalhou com diferentes grupos de alunos através de dois experiências, role playing e concurso. Os resultados obtidos, a partir de um questionário ad-hoc, nos permitiu observar efeitos positivos diretos que tem afetado à melhora na percepção da aprendizagem e da satisfação dos alunos dos cursos onde se aplicou tal metodologia, também esta metodologia tem reforçado o trabalho em equipe dentro de um clima distendido e motivador, incrementando o dinamismo e a interação na aula.

Palavras chave: métodos de aprendizagem, inovação docente, trabalho em equipe, aprendizagem ativa, motivação, universidade, Espanha.

\section{Flipped Classroom Methodology in University Teaching}

\begin{abstract}
In spite of the changes in the habits and behaviour of 21 st century students in terms of interactivity and flexibility, the traditional masterclass methodology still prevails in the university environment; this means that it is necessary to deepen both the application of new methodologies and the students' assessment of their usefulness. In this context, the main objective of this work consists of analyzing the application of the flipped classroom methodology. In order to achieve the planned objective, we have worked with different groups of students by means of two experiences, role playing and competition. The results obtained from an ad-hoc questionnaire have allowed us to observe direct positive effects that have affected the improvement in the perception of learning and student satisfaction of the subjects where it was implemented; besides, this methodology has reinforced teamwork within a relaxed and motivating climate, increasing dynamism and interaction in the classroom.
\end{abstract}

Key words: learning methods, teaching innovation, teamwork, active learning, motivation, university, Spain. 


\section{Introducción}

La inclusión de metodología innovadora en la docencia favorece la obtención de mejores resultados en el aula y en el rendimiento académico del estudiante (Lee, 2011). En concreto, respecto de la docencia en el campo de marketing, las investigaciones (Orus et al., 2014) confirman que los estudiantes que participaron en metodologías innovadoras incrementaron la adquisición de las competencias transversales y mejoraron sus resultados académicos. También en otras áreas de la formación (Bishop y Verleger, 2013) se determinó que los estudiantes están más motivados y tienen una mejor opinión de las asignaturas que aplican dichas metodologías, aunque también existen alumnos reticentes a las mismas.

Siguiendo esta línea de investigación, en este proyecto $^{1}$ hemos implementado la metodología flipped classroom como una innovación docente que ayuda a mejorar la experiencia de aprendizaje de los estudiantes universitarios (Butt, 2014; Mok, 2014). Hasta ahora esta metodología, relativamente nueva, únicamente ha sido tenida en cuenta desde un punto de vista teórico, pero muy pocas investigaciones han basado sus resultados en un estudio empírico donde se analizace la participación activa, dinámica e interactiva del estudiante, la mejora en el aprendizaje de la asignatura y el reforzamiento del trabajo en grupo en un entorno motivador y competitivo.

Se ha implementado en la enseñanza y aprendizaje de tres asignaturas del grado de Administración y dirección de empresas en los cursos de tercero y cuarto: Marketing, Estrategias de marketing y Gestión comercial y ventas. La metodología de investigación utilizada se ha dividido en dos fases: a) unas dinámicas de grupo realizadas con alumnos pertenecientes a las tres asignaturas. El objetivo era conocer cuál era la opinión de los alumnos sobre la aplicación de metodologías innovadoras como flipped classroom y qué esperaban de dichas metodologías; y b) con los resultados de las dinámicas se elaboró un cuestionario que proponía diversas cuestiones a investigar (aprendizaje, satisfacción, interacción, dinamismo, trabajo grupal...), relacionando el role playing y el concurso; la experiencia de los participantes en otras metodologías, y la comparación con las clases tradicionales. Se obtuvo un total de 202 cuestionarios válidos. Los resultados nos han mostrado la eficacia de esta metodología al conseguir efectos positivos directos en la mejora del aprendizaje y de la satisfacción del alumnado de las asignaturas donde se puso en marcha; en el reforzamiento del trabajo en equipo dentro de un clima distendido y motivador; y en el incremento del dinamismo y la interacción en el aula.

Este trabajo se estructura de la siguiente manera: en un primer momento se realizará una revisión de la literatura sobre los conceptos analizados en esta investigación; en segundo lugar, se presenta la metodología utilizada para llevar a cabo la investigación centrándonos en las actividades fipped classroom desarrolladas en las asignaturas implicadas en este estudio; posteriormente se presentarán los resultados, conclusiones, limitaciones y futuras líneas de investigación.

\section{La metodología flipped classroom como innovación en la docencia universitaria}

Pese a los cambios acontecidos en los hábitos y comportamiento de los estudiantes del siglo XXI en términos de interactividad y flexibilidad, en el ámbito universitario sigue predominando la metodología tradicional de clase magistral (Butt, 2014). El proceso de convergencia europea ha supuesto un profundo cambio en el paradigma del proceso

\footnotetext{
${ }^{1}$ Esta investigación se ha realizado en el marco del proyecto de innovación educativa Ref. UV-SFPIE_GER16-417403, Universitat de Valencia. Resolución del 7 de julio de 2016, Vicerrectorat de Politiques de Formació i Qualitat Educativa; y del proyecto de innovación educativa Ref. UV-SFPIE_RMD15-307882, Universitat de Valencia. Resolución del 30 de julio de 2015, Vicerrectorat de Politiques de Formació i Qualitat Educativa..
} 
enseñanza-aprendizaje. Así, los roles, funciones y tareas de los alumnos y de los profesores sufren una importante modificación respecto a la metodología tradicional de clase magistral. Los alumnos deben asumir una postura más activa en el proceso de adquirir los conocimientos necesarios para su formación, mientras que el profesor deberá diseñar las actividades necesarias para que los alumnos puedan aplicar a la resolución de las mismas los conocimientos y capacidades adquiridas de forma más independiente.

En este contexto, que supone trasladar el centro de atención de la enseñanza al aprendizaje, la metodología flipped classroom ha permitido profundizar en estos principios metodológicos del Espacio Europeo de Educación Superior (EEES). Esta metodología invierte el tradicional proceso de enseñanza-aprendizaje, y consiste en proveer a los alumnos de metodologías innovadoras, que les resulten atractivas, como el concurso y el role playing, y que les obliga a buscar previamente los conocimientos teóricos necesarios para poder realizarlas, sin espera a que el profesor las ofrezca en clase, como ocurre con la enseñanza tradicional (Lage et al., 2000).

Uno de los primeros autores que acuñó el término fue Lage (Lage et al., 2000) aunque realmente la expresión no se consolidó como tal hasta 2007 (Bergman y Sams, 2012). Hay autores que plantean que esta metodología se viene utilizando en escuelas de negocio desde hace décadas (Talbert, 2012). Existe abundante literatura sobre la aplicación de esta metodología en el contexto de la educación superior (Berrett, 2012; Tucker, 2012; Ash, 2012) pero es escasa la investigación académica en torno a la metodología flipped classroom en el contexto del marketing.

En nuestra investigación hemos querido comprobar la influencia de diversas variables en los resultados positivos de esta metodología innovadora en la educación superior en marketing. En este sentido, los principales factores a analizar se han centrado:
- En primer lugar, en estudiar si se produce una mayor implicación del estudiante, un aprendizaje más profundo y permite una mayor adaptación al ritmo de cada estudiante (Walsh, 2013),

- En segundo lugar, en analizar si se incrementa la motivación y el rendimiento de los alumnos matriculados en las asignaturas implicadas en esta metodología innovadora (Rosenberg, 2013; Bergam y Sams, 2012; Walsh, 2013; Szoka, 2013).

- En tercer lugar, en investigar si con esta metodología se consigue promover el trabajo colaborativo y los resultados grupales positivos, de manera que permita que los alumnos interactúen entre sí y con el profesor durante el periodo de la enseñanza aprendizaje de las asignaturas de marketing.

- En cuarto lugar, se ha querido investigar si se mejoran los resultados de aprendizaje y la satisfacción de los alumnos al terminar el periodo de formación en marketing.

En definitiva, el objetivo de esta investigación se centraba en probar que el aprendizaje que utiliza metodologías flipped classroom produce mejor trabajo de los alumnos fuera y dentro de clase, lo que se traduce en un aprendizaje mejor consolidado y duradero, un aprendizaje que trasciende la transmisión de conocimientos y facilita el ejercicio y el desarrollo de competencias, un tipo aprendizaje que marcará la diferencia entre el éxito y el fracaso en las instituciones educativas del siglo XXI (Sáez et al., 2014).

\section{Metodología}

Para llevar a cabo nuestra investigación se diseñó un proceso en tres etapas, en función del tipo de información que se deseaba obtener. En primer lugar, se utilizó la técnica de la dinámica de grupo con el objetivo de obtener información de carácter cualitativo 
que nos permitiera diseñar un cuestionario y, a partir de esta fase, proceder a recoger información de carácter cuantitativo. Finalmente se desarrollaba en el aula una actividad práctica innovadora adaptada a cada asignatura.

Para realizar las dinámicas de grupo se invitó a participar, de modo voluntario, a grupos de alumnos de las tres asignaturas implicadas en el estudio (Marketing, Estrategias de marketing, y Gestión comercial y ventas). El objetivo era conocer sus opiniones sobre la aplicación de metodologías innovadoras en la formación universitaria, en concreto, qué les pedirían a esas metodologías y cómo pensaban que deberían aplicarse. Se desarrollaron tres grupos con alumnos de las diferentes asignaturas en cada uno de los mismos. Esta técnica permitió profundizar en sus opiniones y valoraciones más allá de una mera valoración individual, favorecido por el efecto de la interacción que generó el intercambio de opiniones entre los integrantes de cada grupo. Al finalizar la dinámica, el moderador resumía las conclusiones alcanzadas a fin de que los alumnos expresaran su acuerdo, o no, respecto a las mismas y consensuarlas.

A partir de estas conclusiones, y tras una revisión de la literatura (Pérez et al., 2015), el grupo de profesores redactó una serie de ítems que facilitarían el diseño de un cuestionario inicial, posteriormente se volvió a reunir a los alumnos iniciales para que de modo individual analizaran el contenido del mismo en cuanto a su capacidad para reflejar las conclusiones de la dinámica inicial. Tras esta fase, y con unas sencillas modificaciones, se redactó el cuestionario final. En el mismo se solicitaba, en primer lugar, información para clasificar a los alumnos, en concreto el curso de pertenencia, el tipo de actividad innovadora desarrollada y si tenían, o no, experiencia previa en este tipo de actividades innovadoras. En segundo lugar, se les pedía que valorasen la utilidad de las metodologías tradicionales e innovadoras para aprender los contenidos de las diferentes asignaturas (se utilizó una escala tipo Likert de 7 puntos, desde
1 "Nada útil" hasta 7 "Muy útil"). Por último, se les pedía que valorasen para la metodología en la que habían participado una serie de afirmaciones basadas en las conclusiones de las diferentes dinámicas de grupo realizadas, para ello se utilizó una escala tipo Likert de 7 puntos desde 1 "Completamente en desacuerdo" hasta 7 "Completamente de acuerdo".

Como se ha comentado previamente, se utilizaron dos técnicas innovadoras dentro del marco del flipped classroom. En ambos casos, los alumnos trabajaban en grupo y preparaban la actividad de forma previa a la sesión en el aula, por ello debían realizar una recopilación de los contenidos teóricos correspondientes. Por ejemplo, y con base en las indicaciones de la guía de la asignatura, podían buscar contenidos adecuados en internet, en revistas especializadas o incluso acudir a empresas para conocer situaciones reales relacionadas con la materia objeto de análisis.

- Role playing aplicado al marketing. Se trata de simular una situación de la vida diaria y donde cada estudiante integrante de un grupo representa el papel del personaje concreto que le corresponde. El conjunto del grupo representa dicha circunstancia como si se tratara de la realidad, metiéndose individualmente cada uno en su papel, de manera que se interiorice el aprendizaje del tema estudiado y se aprenda a interactuar con la realidad empresarial (por ejemplo, el proceso de venta a un cliente profesional). Esta actividad debía ser grabada en vídeo y, posteriormente, se visionaba y analizaba por todos los alumnos en una de las sesiones de la asignatura.

- Concurso, que se denominó fuego de tronos. El objetivo era conocer todo el contenido de la asignatura de una manera divertida y eficaz. Para ello, se dividió a la clase en subgrupos. Los subgrupos realizaron una lista de preguntas tipo test sobre todos los temas de la asignatura y que fueron revisadas posteriormente por el docente. 
En una segunda fase, se realizó un sorteo para determinar con quién competía cada grupo en la ronda inicial. Se realizaron las rondas eliminatorias, de manera que el subgrupo que obtuvo más aciertos pasó a la ronda siguiente, hasta determinar el subgrupo ganador.

$\mathrm{Al}$ finalizar cada una de las diferentes actividades se solicitaba a los alumnos participantes que complementasen de forma voluntaria y anónima el cuestionario diseñado a partir de las conclusiones de las diferentes dinámicas de grupo realizadas en la primera fase de la investigación. Se obtuvieron un total de 202 cuestionarios válidos, cuyos principales resultados se comentarán más adelante.

Para confirmar poder alcanzar los objetivos de la investigación y analizar las posibles diferencias relacionadas con las variables analizadas, se utilizó una metodología en varias fases (Canales y Hernández, 2015). Todos los análisis se realizaron mediante el programa SPSS (versión 19), utilizando en todos los test realizados un nivel de confianza de al menos 0.05.

En primer lugar, los 26 ítems utilizados en el cuestionario se sometieron a un Análisis Factorial de Componentes Principales (AFCP). En segundo lugar, se realizaron diferentes análisis, fundamentalmente el análisis de diferencias de medias (análisis t y ANOVA) respecto a las puntuaciones otorgadas y el análisis cluster.

El objetivo de este análisis cluster es obtener un conjunto de grupos, en nuestro caso estudiantes, que comparten características similares entre sí y diferentes al resto de grupos. Estos grupos deberán maximizar su homogeneidad interna (intragrupo) y, al mismo tiempo, ser diferentes al resto de grupos, es decir, maximizar su heterogeneidad con el resto de los grupos. El método utilizado ha sido el método de Ward, dentro de los denominados de varianza, y agrupa los individuos de forma jerárquica de modo que minimiza la variación intragrupal de la estructura formada (Mínguez y Fuentes, 2004). Para determinar el número adecuado de grupos, o clusters, analizamos el dendograma y se detectaron tres grupos.

\section{Descripción de la muestra}

Con el objetivo de que los alumnos investigados pudiesen comparar las dos metodologías de formación, tradicionales e innovadoras, se decidió que sólo serían alumnos que ya estuviesen al menos en segundo curso del grado en Administración y Dirección de Empresas. Tal como se muestra en la tabla 1, los grupos mayoritarios son los de tercer y cuarto curso. La mayoría de los alumnos participantes no tenían experiencia previa en las metodologías innovadores. La actividad más realizada fue la de concurso, esta situación se debe a que la actividad se desarrolló en las asignaturas de segundo y tercer curso, mientras que el role-playing se diseñó para una asignatura optativa de cuarto curso.

Respecto a la valoración global que los alumnos otorgan a las dos diferentes metodologías, en una escala de 1 a 7 , podemos indicar que de forma global puntúan con un 5.75 de media a las metodologías innovadoras y con un 4.24 las metodologías tradicionales. Por tanto, podemos intuir que los estudiantes consideran más útil el uso de metodologías innovadores, más participativas, para adquirir los conocimientos necesarios para su formación.

Tabla 1. Descripción de la muestra

\begin{tabular}{|ll|lc|}
\hline \multicolumn{2}{|c|}{ Curso (\%) } & \multicolumn{2}{c|}{ Experiencia previa (\%) } \\
\hline $2^{\circ}$ (Concurso) & 23.8 & Sí & 36.6 \\
$3^{\circ}$ (Concurso) & 38.1 & No & 63.4 \\
$4^{\circ}$ (Role playing) & 38.1 & & \\
\hline
\end{tabular}




\section{Resultados}

\section{Factores de valoración de las actividades}

Tal como se ha comentado anteriormente, en primer lugar, se aplicó un AFCP a los 26 ítems del cuestionario ad-hoc (tabla 2) con el objetivo de agrupar los motivos de valoración de las metodologías aplicadas. Como resultado de este proceso se obtuvieron seis factores (tabla 2) que en conjunto explican un 67.53 de la varianza, siendo su medida de adecuación muestral KMO de 0.843. Además, todos los coeficientes alpha de Cronbach son superiores al valor 0.6, lo cual asegura la consistencia interna de cada uno de los factores resultantes.

El factor 1 (F1), Satisfacción global, recoge cuatro ítems que valoran de forma general la satisfacción con la actividad desarrollada por los alumnos. El segundo factor (F2), Mejora del conocimiento, engloba tres ítems que miden la valoración sobre el aprendizaje y conocimiento de la asignatura en la que se desarrolló la actividad. El tercer factor (F3),
Participación activa, está formado por tres ítems que representan la valoración de los alumnos sobre su grado de participación. El cuarto factor (F4), Interacción, lo forman tres ítems que miden la relación generada entre los alumnos durante la actividad. El factor quinto (F5), Clima de trabajo, lo forman tres ítems que permiten la valoración de las condiciones de trabajo de cada uno de los grupos en los que se dividió a los alumnos. El sexto factor (F6), Capacidad de trabajo en grupo, lo generan tres ítems relacionados con la valoración individual del sistema de trabajo grupal. El séptimo factor (F7), Experiencia personal, lo forman tres ítems que miden el grado de enriquecimiento personal de esta experiencia. El octavo factor (F8), Motivación, está formado por dos ítems relativos a la motivación personal del alumno respecto a la asignatura. El último factor (F9), Recomendación, lo forman 2 ítems referidos al grado de recomendación de la actividad desarrollada por el alumno.

Tabla 2. Factores principales de valoración de las actividades

\begin{tabular}{|l|l|l|}
\hline & & -Cronbach \\
\hline (F1) Satisfacción global & & $\mathbf{0 . 8 4 5}$ \\
\hline La experiencia ha sido satisfactoria & 0.794 & \\
\hline La organización de la actividad ha sido adecuada & 0.749 & \\
\hline Me ha gustado la asignatura & 0.662 & \\
\hline Ha resultado una actividad divertida & 0.644 & \\
\hline (F2) Mejora del conocimiento & & $\mathbf{0 . 7 8 7}$ \\
\hline He mejorado mis conocimientos sobre la materia & 0.748 & \\
\hline He visto la aplicabilidad de los contenidos & 0.702 & \\
\hline Me siento preparado para aplicar lo estudiado & 0.678 & \\
\hline
\end{tabular}




\begin{tabular}{|c|c|c|}
\hline (F3) Participación activa & & 0.701 \\
\hline Me ha permitido conocer la realidad de la asignatura & 0.815 & \\
\hline Es una forma mejor de aprender contenidos teóricos & 0.814 & \\
\hline Facilita el autoaprendizaje & 0.784 & \\
\hline (F4) Interacción & & 0.694 \\
\hline Facilita la relación con el resto de compañeros & 0.821 & \\
\hline Es una forma diferente de ver una asignatura & 0.774 & \\
\hline Genera una relación activa entre los alumnos & 0.718 & \\
\hline (F5) Clima de trabajo & & 0.708 \\
\hline Me he sentido bien con mis compañeros & 0.730 & \\
\hline El ritmo de trabajo ha sido adecuado & 0.695 & \\
\hline Volvería a repetir con el mismo equipo & 0.684 & \\
\hline (F6) Capacidad de trabajo en grupo & & 0.694 \\
\hline He mejorado mi capacidad de relación con otros & 0.800 & \\
\hline Es mejor trabajar en grupo & 0.753 & \\
\hline Hay que escuchar otras opiniones & 0.633 & \\
\hline (F7) Experiencia personal & & 0.672 \\
\hline He descubierto cosas de mí que desconocía & 0.791 & \\
\hline Creo que he mejorado mi forma de trabajar-estudiar & 0.687 & \\
\hline Es aplicable en entornos diferentes al de aprendizaje & 0.686 & \\
\hline (F8) Motivación & & 0.732 \\
\hline Me ha permitido ver la utilidad de los contenidos & 0.732 & \\
\hline Ahora pienso que puede ser una buena actividad profesional & 0.701 & \\
\hline (F9) Recomendación & & 0.659 \\
\hline Es una actividad interesante & 0.699 & \\
\hline Es una actividad recomendable & 0.682 & \\
\hline
\end{tabular}


También se realizó un análisis de correlaciones entre los factores resultantes con el objetivo de detectar la posible existencia de problemas de multicolinealidad entre los mismos. Como se observa en la tabla 3 todos los coeficientes son significativos, por tanto no existen problemas de multicolinealidad entre los datos y cada factor se considera diferente al resto de factores.

Tabla 3. Matriz de correlaciones entre los factores

\begin{tabular}{|c|c|c|c|c|c|c|c|c|c|}
\hline & F1 & F2 & F3 & F4 & F5 & F6 & F7 & F8 & F9 \\
\hline F1 & 1.000 & & & & & & & & \\
\hline $\mathrm{F} 2$ & $0.579^{*}$ & 1.000 & & & & & & & \\
\hline F3 & $0.602^{*}$ & $0.500^{*}$ & 1.000 & & & & & & \\
\hline F4 & $0.585^{*}$ & $0.421^{*}$ & $0.502^{*}$ & 1.000 & & & & & \\
\hline F5 & $0.534^{*}$ & $0.452^{*}$ & $0.395^{*}$ & $0.522^{*}$ & 1.000 & & & & \\
\hline F6 & $0.343^{*}$ & $0.492^{*}$ & $0.399^{*}$ & $0.370^{*}$ & $0.445^{*}$ & 1.000 & & & \\
\hline F7 & $0.322^{*}$ & $0.437^{*}$ & $0.326^{*}$ & $0.275^{*}$ & $0.294^{*}$ & $0.541^{*}$ & 1.000 & & \\
\hline F8 & $0.422^{*}$ & $0.568^{*}$ & $0.419^{*}$ & $0.350^{*}$ & $0.392^{*}$ & $0.501^{*}$ & $0.727^{*}$ & 1.000 & \\
\hline F9 & $0.635^{*}$ & $0.521^{*}$ & $0.523^{*}$ & $0.496^{*}$ & $0.439^{*}$ & $0.356^{*}$ & 0.427 & $0.550^{*}$ & 1.000 \\
\hline
\end{tabular}

${ }^{*}$ Correlaciones significativas $p<0.01$

\section{Valoración de las metodologías innovadoras}

Con el objetivo de conocer en qué grado se alcanzan las diferentes utilidades de las metodologías innovadores indicadas por los alumnos durante las dinámicas de grupo, se les pedía que valoraran aquella en la que habían participado (role playing o concurso). Para determinar la existencia, o no, de diferencias en las valoraciones medias, se utilizó el análisis Prueba t para muestras independientes.

Como se observa en la tabla 4, las metodologías innovadoras son bastante bien valoradas y aceptadas por los alumnos encuestados, en general la valoración se encuentra por encima del cinco. Si comparamos los resultados en función de la actividad concreta realizada por los alumnos, encontramos resultados muy similares en ambas, pues sólo existe una diferencia significativa, en concreto respecto a la valoración de la mejora de la Capacidad de trabajo en equipo. Esta diferencia entre las valoraciones según la experiencia realizada, puede deberse a que, por las características del role playing, no era posible realizarlo de modo individual y posteriormente unir el trabajo en un único video final, mientras que en el concurso se podía trabajar de forma independiente y unirlo al final en una única propuesta.

Otro resultado remarcable es que el aspecto menos valorado se refiere a la capacidad de 
enriquecimiento personal de las actividades, no obstante, el valor medio es alto (4.72). Este resultado puede deberse, seguramente, a que se separa lo puramente personal de la adquisición de conocimientos relacionados con la futura actividad profesional del alumno, y no valoran suficientemente la utilidad de esta nueva metodología para su futuro desarrollo personal y profesional.

Tabla 4. Valoración de la actividad innovadora

\begin{tabular}{|l|c|c|c|}
\hline & Global & Concurso & Role playing \\
\hline Satisfacción global (F1) & 5.56 & 5.56 & 5.57 \\
\hline Mejora del conocimiento (F2) & 5.26 & 5.07 & 5.31 \\
\hline Participación activa (F3) & 5.72 & 5.46 & 5.79 \\
\hline Interacción (F4) & 5.39 & 5.63 & 5.33 \\
\hline Clima de trabajo (F5) & 5.57 & 5.54 & 5.58 \\
\hline Capacidad de trabajo en grupo (F6) * & 5.30 & 4.93 & 5.39 \\
\hline Recomendación (F9) & 5.76 & 5.76 & 5.76 \\
\hline Motivación (F8) & 4.64 & 4.32 & 4.72 \\
\hline
\end{tabular}

Escala desde 1 "Completamente en desacuerdo" hasta 7 "Completamente de acuerdo".

* Diferencia significativa, $p<0.05$, entre tipos de actividad.

\section{Valoración en función del curso}

Una de las primeras cuestiones que queríamos analizar era si con el transcurso del tiempo de formación universitaria cambia la valoración del alumno respecto a las metodologías de aprendizaje con las que interactúa. Para dar respuesta a esta duda, realizamos un análisis ANOVA de diferencias de medias en función del curso en el que se encontraba el alumno encuestado.

Respecto a las valoraciones globales de los dos grandes métodos (tradicionales e innovadores), y pese a que las diferencias no son significativas, hemos de destacar que son los alumnos con menos tiempo en la formación universitaria (los de segundo curso) los que menos valoran la utilidad de las metodologías tradicionales y más valoran las innovadoras. Esto puede ser interpretado como una oportunidad para los profesores a la hora de implantar nuevas metodologías de aprendizaje en el aula (tabla 5).

Respecto a la valoración de la actividad innovadora realizada, lógicamente son los alumnos de segundo curso los que otorgan en general mejores valoraciones a las mismas, aunque existen pocas diferencias significativas entre los tres cursos. Y los que valoran menos las innovaciones son los de tercer curso. 
Tabla 5. Valoración por curso del encuestado

\begin{tabular}{|l|c|c|c|}
\hline & $\mathbf{2}^{\mathbf{0}}$ & $\mathbf{3}^{\mathbf{0}}$ & $\mathbf{4}^{\mathbf{0}}$ \\
\hline Metodología tradicional & 3.98 & 4.45 & 4.19 \\
\hline Metodología innovadora & 6.02 & 5.61 & 5.71 \\
\hline Satisfacción global (F1) & 5.69 & 5.52 & 5.53 \\
\hline Mejora del conocimiento (F2) & $5.94^{*}$ & 4.88 & 5.22 \\
\hline Participación activa (F3) & 5.94 & $5.48^{*}$ & 5.83 \\
\hline Interacción (F4) & 5.31 & 5.57 & 5.26 \\
\hline Clima de trabajo (F5) & 5.52 & 5.47 & 5.71 \\
\hline Capacidad de trabajo en grupo (F6) & 5.73 & $4.82^{*}$ & 5.51 \\
\hline Experiencia personal (F7) & 4.90 & 4.32 & 4.79 \\
\hline Motivación (F8) & 5.33 & 4.90 & 5.14 \\
\hline Recomendación (F9) & 5.94 & 5.65 \\
\hline
\end{tabular}

Escala desde 1 "Completamente en desacuerdo" hasta 7 "Completamente de acuerdo".

* Diferencia significativa respecto a los otros dos cursos, $p<0.05$.

\section{Valoración en función de la experiencia previa}

Otra de las cuestiones a analizar consistía en conocer si el haber participado anteriormente en actividades de formación innovadora influía, o no, en la valoración de la utilidad de las mismas. Como se muestra en la tabla 6 , en general los alumnos con experiencia previa en este tipo de actividades valoran más positivamente la realización de las mismas, lo que puede significar que con el uso de las mismas por parte de los profesores se mejora la capacidad de los alumnos para beneficiarse de ellas.
Existen diferencias significativas en cuatro de las nueve afirmaciones, y todas estas hacen referencia a la utilidad de las metodologías más allá de las asignaturas concretas en la que se apliquen. Por tanto, podemos pensar que para los alumnos encuestados la aplicación de metodologías innovadoras mejora su proceso de aprendizaje. En general, podemos indicar que los estudiantes universitarios han percibido la aplicación de nuevas metodologías de aprendizaje como valiosas e interesantes para mejorar su proceso de formación y adquisición de habilidades para aplicar a la realidad los conocimientos teóricos de las diferentes asignaturas. 
Tabla 6. Valoración en base a la experiencia previa

\begin{tabular}{|l|c|c|}
\hline & sí & NO \\
\hline Satisfacción global (F1) & 5.58 & 5.55 \\
\hline Mejora del conocimiento (F2)* & 5.51 & 5.12 \\
\hline Participación activa (F3) & 5.85 & 5.65 \\
\hline Interacción (F4) & 5.41 & 5.38 \\
\hline Clima de trabajo (F5) & 5.61 & 5.55 \\
\hline Capacidad de trabajo en grupo (F6) & 5.51 & 5.17 \\
\hline Expecomendación (F9)* & 5.89 & 4.93 \\
\hline Motivación (F8)* & & \\
\hline & & \\
\hline
\end{tabular}

Escala desde 1 "Completamente en desacuerdo" hasta 7 "Completamente de acuerdo". * Diferencia significativa, $p<0.05$.

\section{Análisis clúster: agrupación de alumnos}

Realizamos un análisis con el objetivo de agrupar a los alumnos en función de su mayor o menor aceptación de las metodologías innovadoras. Para ello realizamos, en primer lugar, un análisis cluster que nos permitiera determinar la existencia de grupos con comportamientos diferentes hacia las nuevas metodologías. Para realizar este análisis se utilizaron las dos preguntas relativas a la valoración global de las metodologías tradicionales e innovadoras. Posteriormente realizamos el análisis adecuado para determinar dichas diferencias.

A fin de determinar el número de grupos, o cluster, analizamos en dendograma y determinamos la existencia de tres grupos diferenciados, cuyas valoraciones medias respecto a la utilizada de las mencionadas metodologías se muestran en la tabla 7.
Del análisis de los resultados obtenidos podemos describir a los tres grupos. El grupo 1 está formado por los alumnos que prefieren las metodologías innovadoras y que no consideran útil la formación basada en sistemas tradicionales, por lo que podemos denominarlos alumnos innovadores, y son el grupo mayoritario (56.9\% de la muestra). En el grupo 2 se ubican aquellos alumnos (30.7\% de la muestra) que presentan valores altos en ambas metodologías y, por tanto, los podemos clasificar como indecisos en base a nuestro objetivo de clasificación. Por último, el grupo 3 lo integran aquellos alumnos que no valoran las metodologías innovadoras y que prefieren ligeramente los sistemas tradicionales, los podemos clasificar como alumnos reacios, afortunadamente son la minoría (12.4\% de la muestra). Por último, y con el fin de caracterizar a los tres grupos y detectar 
las posibles diferencias entre ellos, realizamos un análisis ANOVA, cuyos resultados se muestran en la tabla 8.

Como se puede observar los grupos 1 (innovadores) y 2 (indecisos) valoran muy positivamente el uso de metodologías innovadores en su formación universitaria, y únicamente presentan diferencias respecto al enriquecimiento como persona que les proporciona el método (aunque en ambos casos es elevado). Por su parte, el grupo 3 (reacios), presenta todos sus valores inferiores y estadísticamente diferentes a los otros dos grupos y parece que las metodologías innovadoras no les aportan nada interesante para su formación universitaria.

Tabla 7. Valoración por agrupación de alumnos encuestados

\begin{tabular}{|l|c|c|c|}
\hline & Grupo 1 & Grupo 2 & Grupo 3 \\
\hline Metodología tradicional & 3.50 & 5.55 & 4.44 \\
\hline Metodología innovadora & 5.90 & 6.32 & 3.60 \\
\hline
\end{tabular}

Tabla 8. Valoración de la metodología innovadora por grupo

\begin{tabular}{|l|c|c|c|}
\hline & G 1 & G 2 & G 3 \\
\hline Satisfacción global (F1) & 5.72 & 5.71 & $4.48^{*}$ \\
\hline Mejora del conocimiento (F2) & 5.46 & 5.50 & $3.76^{*}$ \\
\hline Participación activa (F3) & 5.90 & 5.77 & $4.76^{*}$ \\
\hline Interacción (F4) & 5.49 & 5.50 & $4.68^{*}$ \\
\hline Clima de trabajo (F5) & 5.59 & 5.79 & $4.96^{*}$ \\
\hline Capacidad de trabajo en grupo (F6) & 5.41 & 5.52 & $4.24^{*}$ \\
\hline Experiencia personal (F7) & 5.88 & 6.06 & $4.48^{*}$ \\
\hline Motivación (F8) & $5.58^{*}$ & 5.11 & $3.72^{*}$ \\
\hline
\end{tabular}

Escala desde 1 "Completamente en desacuerdo" hasta 7 "Completamente de acuerdo".

* Diferencia significativa respecto a los otros dos grupos, $p<0.05$. 


\section{Conclusiones, limitaciones y futuras líneas de investigación}

Como conclusión de este estudio inicial, podemos destacar la positiva valoración que hacen la mayoría de los alumnos encuestados de la aplicación de metodologías innovadoras para mejorar su proceso de aprendizaje universitario. Esta conclusión está en sintonía con otros estudios anteriores, aunque en nuestro caso se ha centrado en dos métodos concretos y diferentes entre sí como son el concurso y el role playing.

También hay que destacar que son los alumnos con menos tiempo en la universidad los que, en general, valoran mejor las nuevas metodologías y que, por tanto, los profesores podemos y debemos utilizar esta predisposición para desarrollar e implantar nuevas formas de aportarles conocimiento, y no sólo utilizar lo tradicional.

Los alumnos con experiencia previa en este tipo de actividades formativas responden más positivamente a las mismas, lo cual parece ratificar la utilidad de las mismas y su aceptación por parte del alumnado.

No obstante, no todos los alumnos consideran positivo ese tipo de innovaciones y existen, aunque son minoría, aquellos que son reacios a la incorporación de nuevas formas para recibir su formación universitaria. La gran mayoría del alumnado valora positivamente la implantación de actividades innovadores que les permitan una mayor participación y ser más activos en su formación, sin descartar los métodos más tradicionales.

En definitiva, aunque la percepción que tienen los estudiantes del empleo de esta metodología integrada por el concurso y el role playing, es que resulta útil e interesante, no obstante, han confirmado que el uso de esta metodología requiere más trabajo y esfuerzo por parte de los estudiantes. Sin embargo, tanto los resultados académicos obtenidos en esta actividad, como en los resultados obtenidos en la prueba final integradora (concurso y role playing), se observa cómo el aprendizaje de estas asignaturas de marketing ha sido más profunda.

También sería conveniente en la sesión introductoria de lo que es una flipped classroom, mostrar a los alumnos que van a realizar la experiencia, las evidencias obtenidas con otros grupos en la mejora de los resultados empleando esta metodología. De esta forma se podría motivar a los que más resistencia oponen al cambio del proceso enseñanza-aprendizaje.

En cuanto a las limitaciones de esta investigación, como ya hemos indicado se trata de una primera aproximación al tema, en la que analiza la opinión o valoración personal de los encuestados y no los resultados obtenidos por los alumnos en las asignaturas implicadas. La muestra sólo está conformada por alumnos de una carrera, el Grado de Administración y Dirección de Empresas de una universidad. Tampoco se han utilizado escalas previamente validadas, pues uno de los objetivos ha sido conocer qué demandan los alumnos universitarios a las nuevas metodologías. Además, sólo se ha trabajado con dos técnicas, el concurso y el role playing dentro de la gran variedad de metodologías de aprendizaje flipped classroom. Por tanto, estas limitaciones se convierten en futuras líneas de investigación que tendrán como objetivo mejorar tanto la representatividad de la muestra dentro del ámbito universitario como las herramientas utilizadas para recoger la opinión de los alumnos (escalas validadas) y la aplicación de otras metodologías participativas. Por último, también nos proponemos conocer la opinión y valoración de los profesores respecto a la implantación de estas metodologías activas, así también sería deseable poder analizar las posibles diferencias en cuanto a los conocimientos adquiridos entre el uso de metodologías tradicionales e innovadoras. 


\section{Referencias}

Ash, Katie (2012), "Educators evaluate flipped classrooms", en Education Week, vol. 32, núm. 2, pp. 6-8. Bergmann, Jonathan y Aaron Sams (2012), Flip your classroom: reach every student in every class every day, Washington, International Society for Technology in Education.

Berrett, Dan (2012), "How flipping classroom can improve the traditional lecture", en The Chronicle of Higher Education, pp. 19-23.

Bishop, Jacob y Matheu Verleger (2013), "The flipped classroom: A survey of the research", en 120th ASEE Conference Proceedings, Atlanta, <http://www. studiesuccesho.nl/wp-content/uploads/2014/04/ flipped-classroom-artikel.pdf $>$ [Consulta: mayo de 2017].

Butt, Adam (2014), "Student views on the use of a flipped classroom approach: Evidence from Australia", en Business Education and Acaguacreditation, Hilo-Hawai, The Institute for Business \& Finance Research, vol. 6, núm. 1, pp. 33-43.

Canales, Pedro y Asunción Hernández (2015), “Aplicación del Food Choice Questionaire en jóvenes adolescentes y su relación con el sobrepeso y otras variables sociodemográficas", en Nutrición Hospitalaria, Madrid, Grupo Aula Médica, vol. 31, núm. 5, pp. 1968-1976 [DOI:10.3305/nh.2015.31.5.8389].

Lage, Maureen J. et al. (2000), "Inverting the classroom: a gateway to creating an inclusive learning environment", en Fournal of Economic Education, Nebraska, Routledge, vol. 31, núm. 1, pp. 30- 43.

Lee, Yu-Je (2011), "A study on the effect of teaching innovation on learning effectiveness with learning satisfaction as a mediator", en World transaction on engineering and technology education, Melbourne, WIESTE, vol. 9, núm. 2, pp. 92-101.
Mínguez, Andrés y María Fuentes (2004), Cómo hacer una investigación social, Valencia, Ed. Tirant lo Blanch.

Mok, Heng Ngee (2014), "Teaching tip: the flipped classroom", en fournal of information systems education, Wilgmington, EDSIG, vol. 25, núm. 1, pp. 7-11.

Orus, Carlos et al. (2014), "Consecuencias afectivas y objetivas del uso de un canal de Youtube de apoyo a la docencia de marketing", en XXVI Congreso Nacional de Marketing-AEMARK, Madrid, ESIC Editorial.

Pérez, Andrea et al. (2015), "Evaluación de la técnica flipped classroom como innovación de éxito en la docencia universitaria de marketing" en XXVII Congreso de Marketing AEMARK, Madrid, ESIC Editorial.

Rosenberg, Tina (2013), "Turning education upside down", en: <http://opinionator.blogs.nytimes. com/2013/10/09/turning-education-upside-down/> [Consulta: mayo de 2017].

Szoka, James (2013), "Measured results demonstrate enhanced learning outcomes in the flipped classroom", en: <http://www.emergingedtech.com/2013/05/ measuredresults-demonstrate-enhanced-learning-outcomesin-the-flipped-classroom/> [Consulta: mayo de 2017].

Talbert, Robert (2012), "Inverted classroom”, en Colleagues, vol. 9, núm. 1, pp. 7-9.

Tucker, Bill (2012), "The flipped classroom", en Education Next, vol. 12, núm. 1, pp. 82-83.

Sáez, Beatriz y María Piedad Ros (2014), "Una experiencia de fipped classroom", en XI fornadas Internacionales de Innovación Universitaria Educar para transformar.

Walsh, Kelly (2013), "Flipped Classroom Panel Discussion Provides RichInsightsintoaPowerful TeachingTechnique", en: <http://www.emergingedtech.com/2013/06/ flipped-classroom-panel-discussion-provides-rich-insightsinto-a-powerful-teaching-technique/>.

\section{Cómo citar este artículo:}

Canales-Ronda, Pedro y Asunción Hernández-Fernández (2019), "Metodología flipped classroom en la enseñanza universitaria”, en Revista Iberoamericana de Educación Superior (RIES), México, UNAM-IISUE/Universia, vol. x, Núm. 28, pp. 116-130, DOI: https://doi.org/10.22201/iisue.20072872e.2019.28.432 [consulta: fecha de última consulta]. 